\title{
Leaving the loners alone: Evolution of cooperation in the presence of antisocial punishment
}

\author{
Julián García, Arne Traulsen* \\ Evolutionary Theory Group, Max-Planck-Institute for Evolutionary Biology, August-Thienemann-Straße 2, 24306 Plön, Germany
}

\section{H I G H L I G H T S}

- Antisocial punishment does not always hinder the evolution of cooperation.

- Whether cooperation is favored or not depends on how loners interact with others.

- If loners are truly alone, responsible punishment and cooperation do evolve.

\section{A R T I C L E I N F O}

\section{Article history:}

Received 7 February 2012

Received in revised form

8 May 2012

Accepted 10 May 2012

Available online 23 May 2012

Keywords:

Punishment

Cooperation

Collective action

Antisocial punishment

\begin{abstract}
A B S T R A C T
The idea that voluntary participation may promote the evolution of cooperation and punishment in public good games has been recently called into question based on the study of the complete strategy set in which anyone can punish anyone else. If punishment actions are detached from contribution and participation in the game, the combination of punishment and voluntary participation no longer leads to high levels of cooperation. We show that this result crucially depends on specific details of the role of those who abstain from the collective endeavour, and only holds for a small subset of assumptions. If these loners are truly alone, cooperators who punish only defectors prevail, even when antisocial punishment is available.
\end{abstract}

(c) 2012 Elsevier Ltd. All rights reserved.

\section{Introduction}

Large scale cooperation amongst unrelated individuals remains an evolutionary puzzle (Sigmund, 2010). A profusion of interdisciplinary research has been devoted to solve this conundrum in the past years (Sigmund, 2007). Punishment has been shown to be an important part of the puzzle (Yamagishi, 1986; Fehr and Gächter, 2000; Fehr and Gachter, 2002; Boyd et al., 2003; Henrich et al., 2006; Johnson et al., 2009), and the combination of voluntary participation and costly punishment seems to provide an ultimate explanation for cooperation in sizable groups (Fowler, 2005; Hauert et al., 2007; Traulsen et al., 2009). This idea has been refined in a series of papers (Brandt et al., 2006; Hauert et al., 2008; Mathew and Boyd, 2009; De Silva et al., 2010; Helbing et al., 2010), but recent work argues that the possibility of antisocial punishment (i.e., strategies that punish cooperators) compromises the stability of cooperation (Rand and Nowak, 2011; Dreber and Rand, 2012). We show that the evolution of punishment critically depends on the microeconomic assumptions about the role of those who abstain from playing the game. In

\footnotetext{
* Corresponding author. Tel.: +494522 763239.

E-mail addresses: garcia@evolbio.mpg.de (J. García), traulsen@evolbio.mpg.de (A. Traulsen).
}

particular, there are scenarios in which antisocial punishment is possible, yet cooperation and responsible punishment still evolve.

The model of costly punishment and voluntary participation, first discussed by Fowler (2005) and Brandt et al. (2006), considers the following strategies: cooperators $(C)$, who invest a given endowment in a joint enterprise; defectors $(D)$, who do not invest in the public good but benefit from it; punishers, who cooperate and in addition punish those who do not cooperate $(P)$; and loners $(L)$, who get a fixed payoff abstaining from the game. Studying infinite populations without demographic noise leads to bistable outcomes (Brandt et al., 2006), which are resolved by addressing the dynamics in a finite population of size $M$ with small mutation rates (Hauert et al., 2007). At every time step, $n$ individuals are chosen and offered the option to participate in a public goods game. Loners get a fixed payoff $\sigma>0$, and do not participate in the game. Cooperators contribute to the joint enterprise at a cost $c>0$ to themselves, defectors rely on the contributions of others. All contributions are summed up and multiplied by a factor $r$ (where $1<r<n$ ). The resulting quantity is equally divided amongst those who choose to participate in the game. If there is a single participant, then she gets the loner payoff $\sigma$. The joint enterprise is risky. The payoff of participants is zero if everyone defects, and thus loners would be better off. Cooperation is a better option as soon as two players cooperate. However, cooperation is prone to the exploitation of defectors again (Hauert et al., 
2002). In the punishment stage of the game each contributor can impose a fine $\beta$ on defectors, at a personal cost $\gamma$ (strategy $P$ ).

We study evolution in this game by looking at a Moran process with rare mutations and exponential payoff to fitness mapping (Traulsen et al., 2008). The stationary distribution of the associated Markov chain can be computed exactly if mutations are sufficiently small (Fudenberg and Imhof, 2006). We assess the effect of selection and mutation by inspecting strategy abundance, i.e., the frequency of each strategy in the stationary distribution (see Appendix A).

The results of Hauert et al. (2007) show that an evolving population spends a considerable amount of time in cooperative states. Punishers are vulnerable to invasion by cooperators who do not pay the cost of punishing, which in turn are susceptible to invasion of defectors. But loners offer a way out of defection and back into cooperation with or without punishment. This holds if a loner earns a higher payoff than a defector would get in a group of defectors $(\sigma>0)$, but a lower payoff than a cooperator would get in a group of cooperators $(\sigma<c(r-1))$ (Hauert et al., 2007; Mathew and Boyd, 2009). Even though loners do not have a large share of the frequencies in the long run, their presence is essential to maintain cooperation. Thus, the system evolves via freedom to coercion (see Appendix B).

The strategy set of Hauert et al. (2007) contains only four strategies. It assumes that only those who cooperate can punish and that punishment is exclusively targeted at defectors. Under neutrality, cooperative actions would be overrepresented because they are associated with two strategies $(C$ and $P$ ). Moreover, as noted by Rand and Nowak (2011), "there is no reason to assume a priori that only cooperators punish others". Evidence also suggest that human subjects can exhibit antisocial punishment (i.e., punishment directed at cooperators) (Herrmann et al., 2008; Rand et al., 2010), a type of strategy that is not considered in this simple setting.

In a recent model Rand and Nowak (2011) detach the punishment option from any particular strategy. Individuals can contribute to the
A
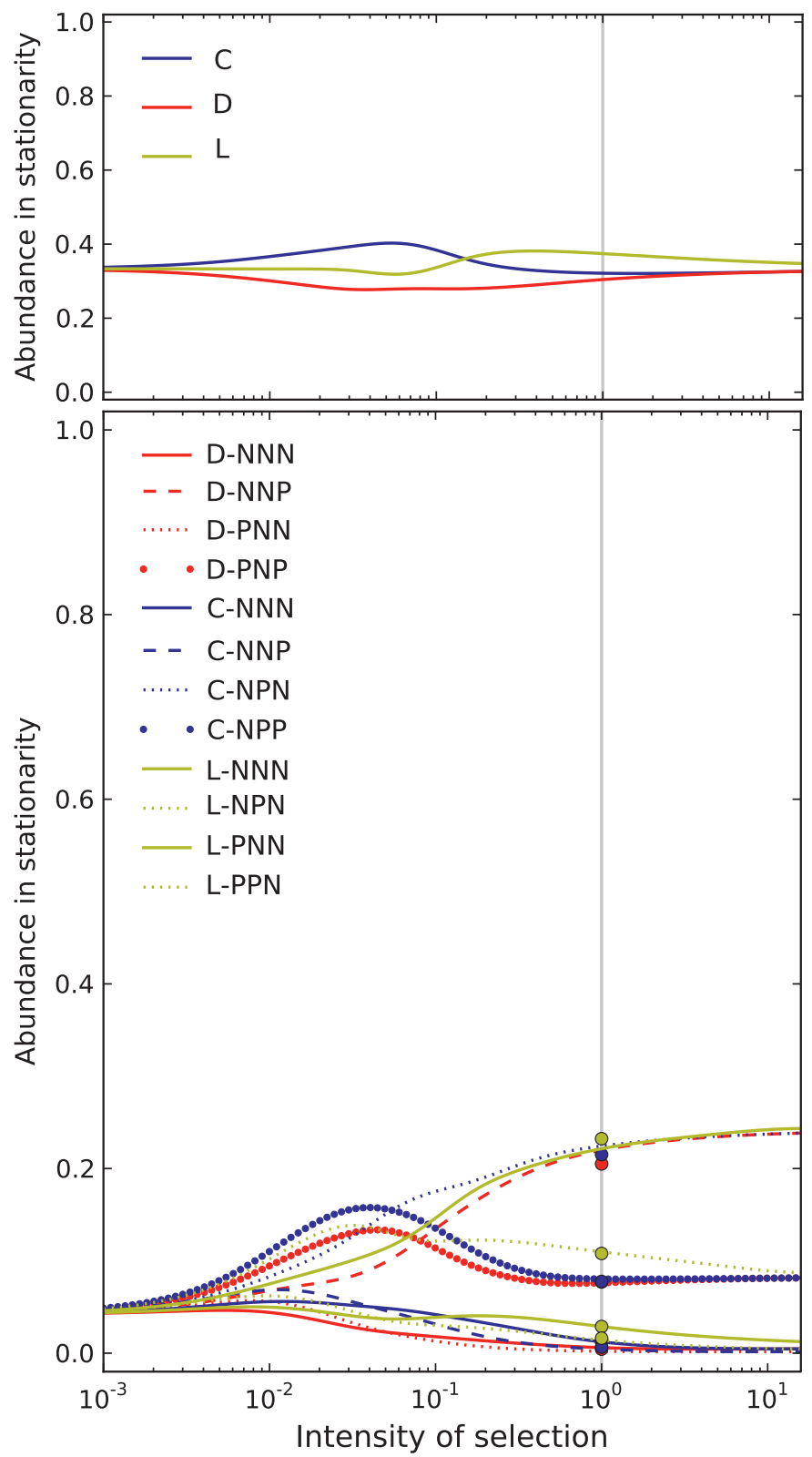

B

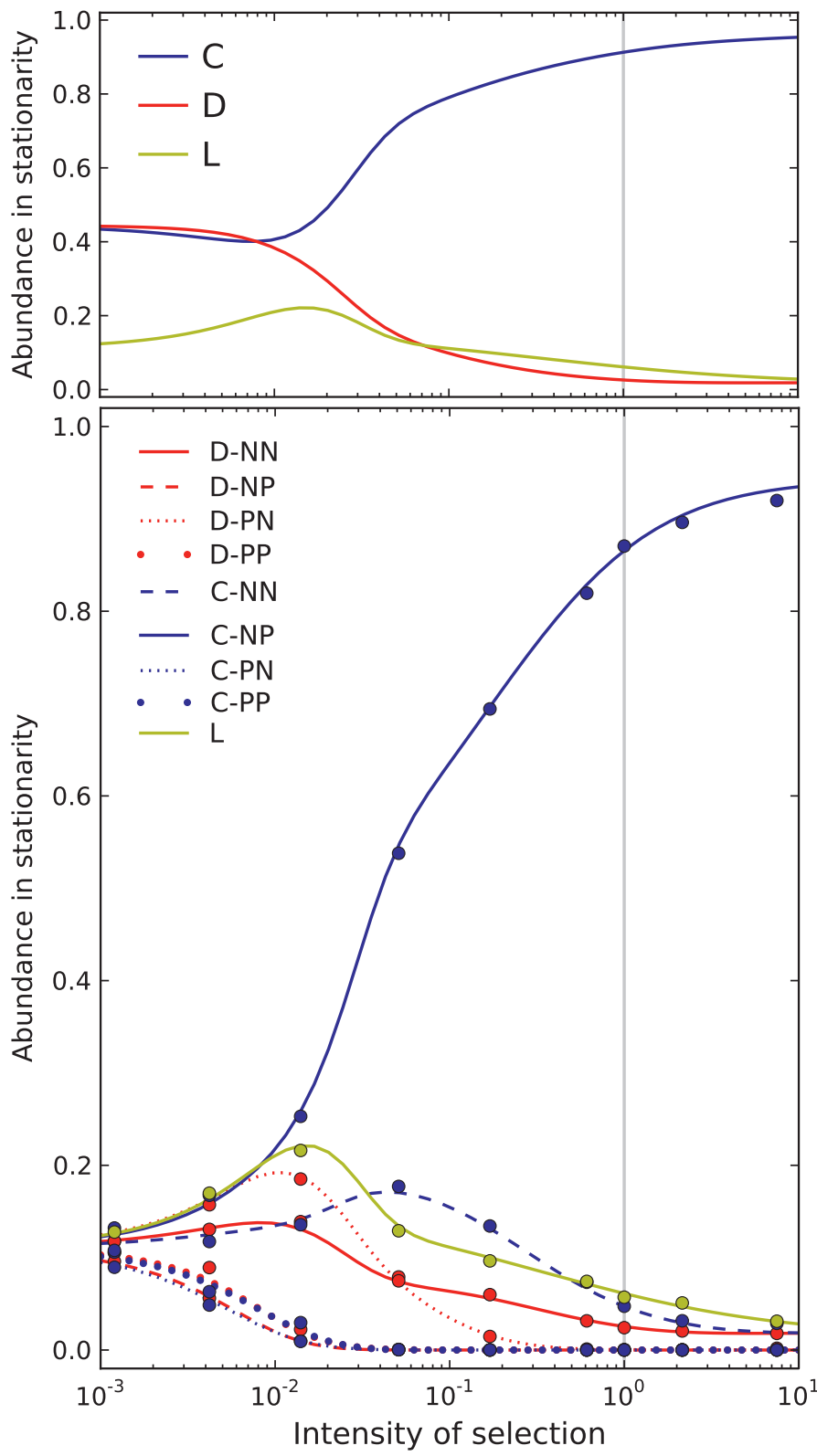

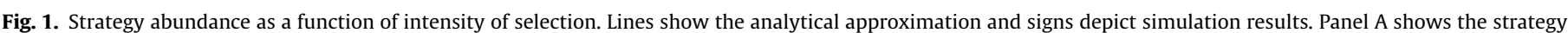

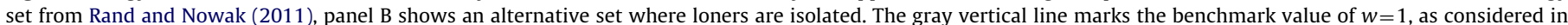

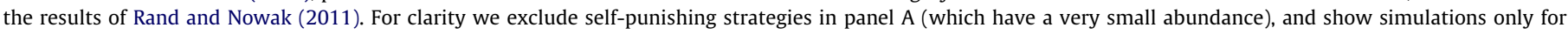
$w=1$. In the upper part, strategies are grouped by their contribution to the public good. Parameters are: $M=100, n=5, r=3, \gamma=0.3, \beta=\sigma=c=1$. 
public good game $(C)$, play avoiding contribution $(D)$ or abstain from playing $(L)$. In addition, they can decide whether or not to punish each of the other types. A strategy is then a 4-tuple $\left[a_{1}, a_{2}, a_{3}, a_{4}\right]$, where $a_{1}=D, C$ or $L$, and $a_{2}, a_{3}, a_{4}=P$ or $N$. Element $a_{1}$ codes for defection, contribution, or abstention; $a_{2}$ determines whether to punish cooperators, $a_{3}$ determines whether to punish defectors, and $a_{4}$ determines whether to punish loners. It is a assumed that an individual never punishes herself, but may punish others using the same strategy. The strategy set is composed of $3 \times 2 \times 2 \times 2=24$ strategies.

This strategy set is appealing for two reasons. First, it is "fair" in the sense that under neutrality, neither cooperative nor uncooperative actions are favored; and second, it does not restrict the punishment option to cooperators, and thus allows for antisocial punishment. The results of Rand and Nowak (2011) are replicated in panel A of Fig. 1. The most popular strategies are cooperators who punish defectors, defectors who punish loners, and loners who punish cooperators. In the limit of strong selection and large populations, the abundance of $D-N N P, C-N P N$ and $L-P N N$ converges, each, to $\frac{1}{4}$; the abundance of strategies $D-P N P, C-N P P$ and $L-P P N$ approaches $\frac{1}{12}$, and all other strategies are not present. For any intensity of selection, no strategy is more abundant than $\frac{1}{4}$.

Here we have two remarkable outcomes. First, evolution favors punishment targeted at cooperators (mostly by loners) as much as it favors punishment targeted at any other strategy. Second, there is a large share of loners and defectors in the long run, challenging the "commonly held view that punishment promotes the evolution of cooperation" (Rand and Nowak, 2011).

\section{Leaving the loners alone}

While there is no reason to assume a priori that only cooperators can punish only defectors (Rand and Nowak, 2011), in many situations it is reasonable to assume that loners cannot punish or be punished (see Discussion). We therefore consider an alternative strategy set, where loners are isolated. Individuals can contribute to the public good game $(C)$, play avoiding contribution $(D)$ or abstain from playing $(L)$. In addition, cooperators and defectors can decide whether to punish or not other cooperators or defectors. The strategy set is then composed of all triplets $\left[a_{1}, a_{2}, a_{3}\right]$, where $a_{1}=D, C$, and $a_{2}, a_{3}=P$ or $N$. Element $a_{1}$ codes for contribution in the public goods game, $a_{2}$ determines whether or not to punish cooperators, $a_{3}$ determines whether or not to punish defectors. In addition to the triplets, we have the loner strategy. The whole set is composed of $2 \times 2 \times 2+1=9$ strategies. This subset has the same two appealing features of the set considered by Rand and Nowak - in particular, it allows the evolution of antisocial punishment - but it excludes loners from punishment. It is also fair, in the sense that neither cooperation nor defection are favored in neutrality conditions.

The outcome of evolution acting on this subset of strategies is shown in panel B of Fig. 1. Here, it is clear that strategy $C-N P$, i.e., cooperators who punish only defectors, prevails for sufficiently strong selection. This strategy is equivalent to $P$ in the model of Hauert et al. (2007). For this subset of nine strategies we can calculate the stationarity distribution in the limit of strong selection. It is given by

$\left(\begin{array}{c}x_{D-N N} \\ x_{D-N P} \\ x_{D-P N} \\ x_{D-P P} \\ x_{C-N N} \\ x_{C-N P} \\ x_{C-P N} \\ x_{C-P P} \\ x_{L}\end{array}\right)=\frac{1}{8+M}\left(\begin{array}{c}2 \frac{M+1}{M+2} \\ 0 \\ \frac{2}{M+2} \\ 0 \\ 2 \\ M+2 \\ 0 \\ 0 \\ 2\end{array}\right)$

where $x_{S}$ denotes the relative abundance of strategy $S$, such that $\sum_{S} x_{S}=1$.
The four strategies that punish others of their own type have abundance zero (i.e., $D-N P, D-P P, C-P N, C-P P$ ). Moreover, from the stationary distribution we note that the inequality

$x_{C-N P}>x_{C-N N}=x_{L}>x_{D-N N}>x_{D-P N}$

holds for any $M$, which means that cooperators who punish defectors are favored over defectors who punish cooperators. If we let the population size go to infinity $(M \rightarrow \infty)$, cooperators who punish only defectors prevail, i.e., $x_{C-N P} \rightarrow 1$, and the abundance of other strategies approaches zero. This is structurally the same result obtained with the restricted strategy set of Hauert et al. (2007) (see Appendix Appendix B). Therefore, assuming that loners are truly alone, we recover the results of the simpler, and maybe more intuitive model, where only four strategies are considered a priori.

The model by Rand and Nowak (2011) shows that punishment targeted at all other strategies can intercept the cyclic dominance in Hauert et al. (2007) (see Appendix B). In the model with the complete strategy set, the most popular strategies are locked in a cycle of spite-you should punish the dominant type to stabilize your own. Therefore $C$ punishes $D, D$ punishes $L$ and $L$ punishes $C$. These strategies have a similar abundance, and as it can be seen in the upper part of Fig. 1A, lead to a situation in which selection does not favor any particular contribution action. The abundance of cooperative, defective and abstaining strategies is almost constant for any intensity of selection. When loners are excluded from punishment the cyclic dominance is re-established and there is strong selection pressure for contributing to the public good (see the upper part of Fig. 1B).

\section{Partial isolation of loners}

In order to understand how punishment, from and towards loners, affects the outcome of evolution, we analyzed the two additional situations in which loners are only partially isolated.

\subsection{Loners cannot punish}

First, loners are unable to punish, yet they can be punished by cooperators and defectors. With this subset we have $2 \times$ $2 \times 2 \times 2+1=17$ strategies, as described in Table $\mathrm{C} 1$ in the appendix. This leads to essentially the same outcome obtained with the full set of 24 strategies, with the exception that loners who punish others are not present because they are excluded ex ante. If loners can be punished, punishment fails to promote cooperation, which is just as equally represented as defection in the long run for strong selection intensity (see Panel A, of Fig. 2). In other words, the results are similar to those of Rand and Nowak.

When loners can be punished, yet they cannot punish others (Fig. 2A) the stationary distribution in the limit of strong selection is

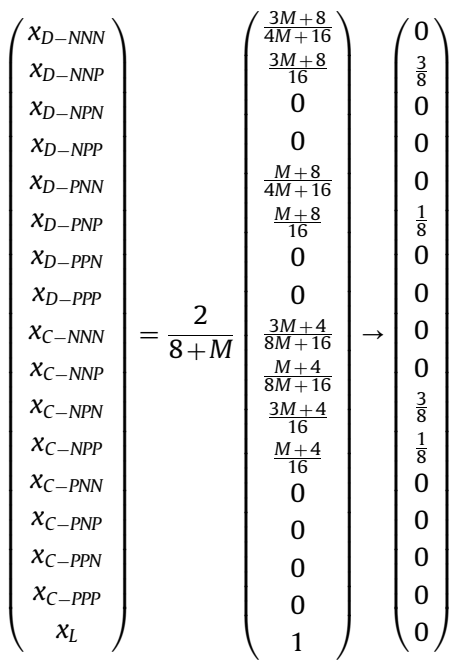


where the limit is for $M \rightarrow \infty$. In this case, we have, $x_{D-N N P}=$ $x_{C-N P N}=\frac{3}{8}, x_{D-P N P}=x_{C-N P P}=\frac{1}{8}$; all other strategies have abundance zero. This means that cooperative strategies are as equally represented as defective strategies when selection is strong and the population size is large. In this setup, punishment does not promote cooperation, despite the option to abstain.

\subsection{Loners cannot be punished}

Now consider the situation in which loners are able to punish cooperators and defectors, but cannot be punished. In this subset we have $3 \times 2 \times 2=12$ strategies, as described in Table C2 in the appendix. As in the model with completely isolated loners, cooperators who punish defectors are highly represented in the stationary distribution when selection is not weak (see Panel B of Fig. 2). In this case, we qualitatively recover the results of Hauert et al. (2007).

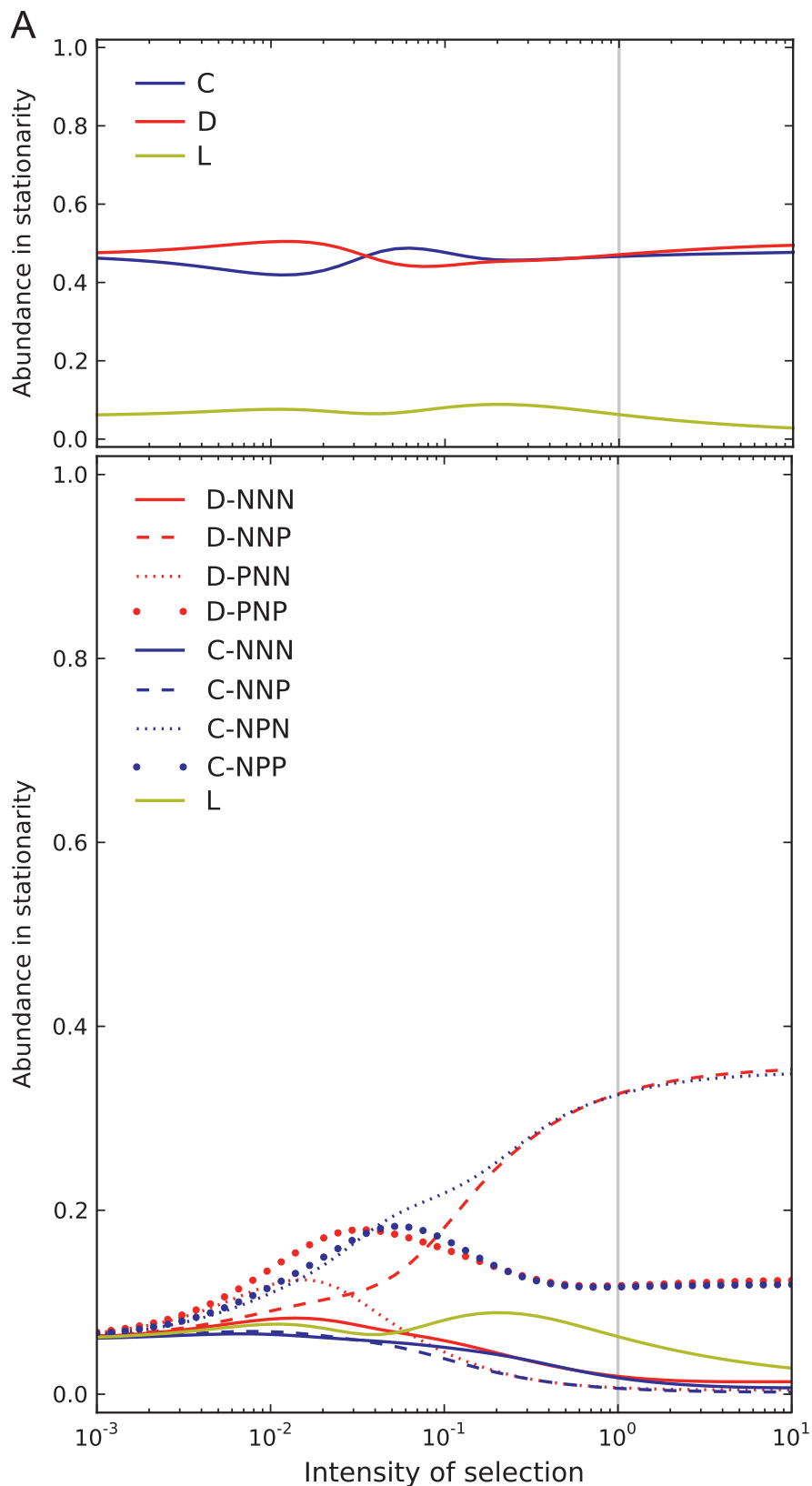

When loners cannot be punished, but can punish others; the stationary distribution in the limit of strong selection is

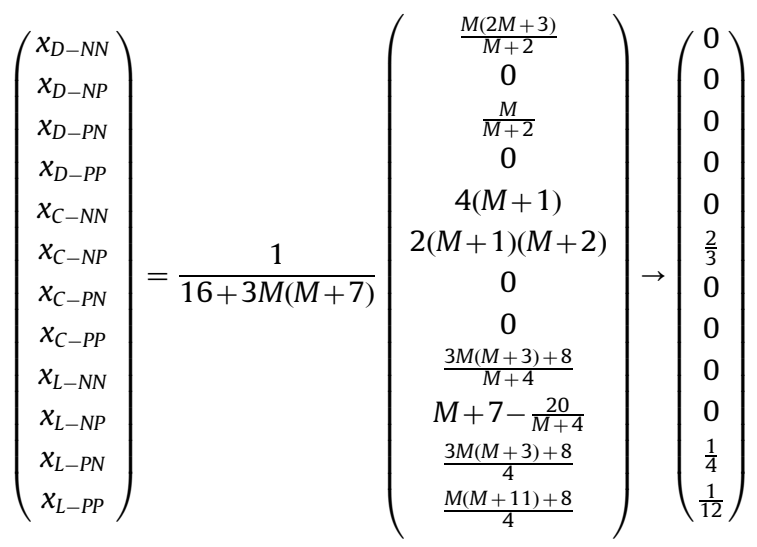

B
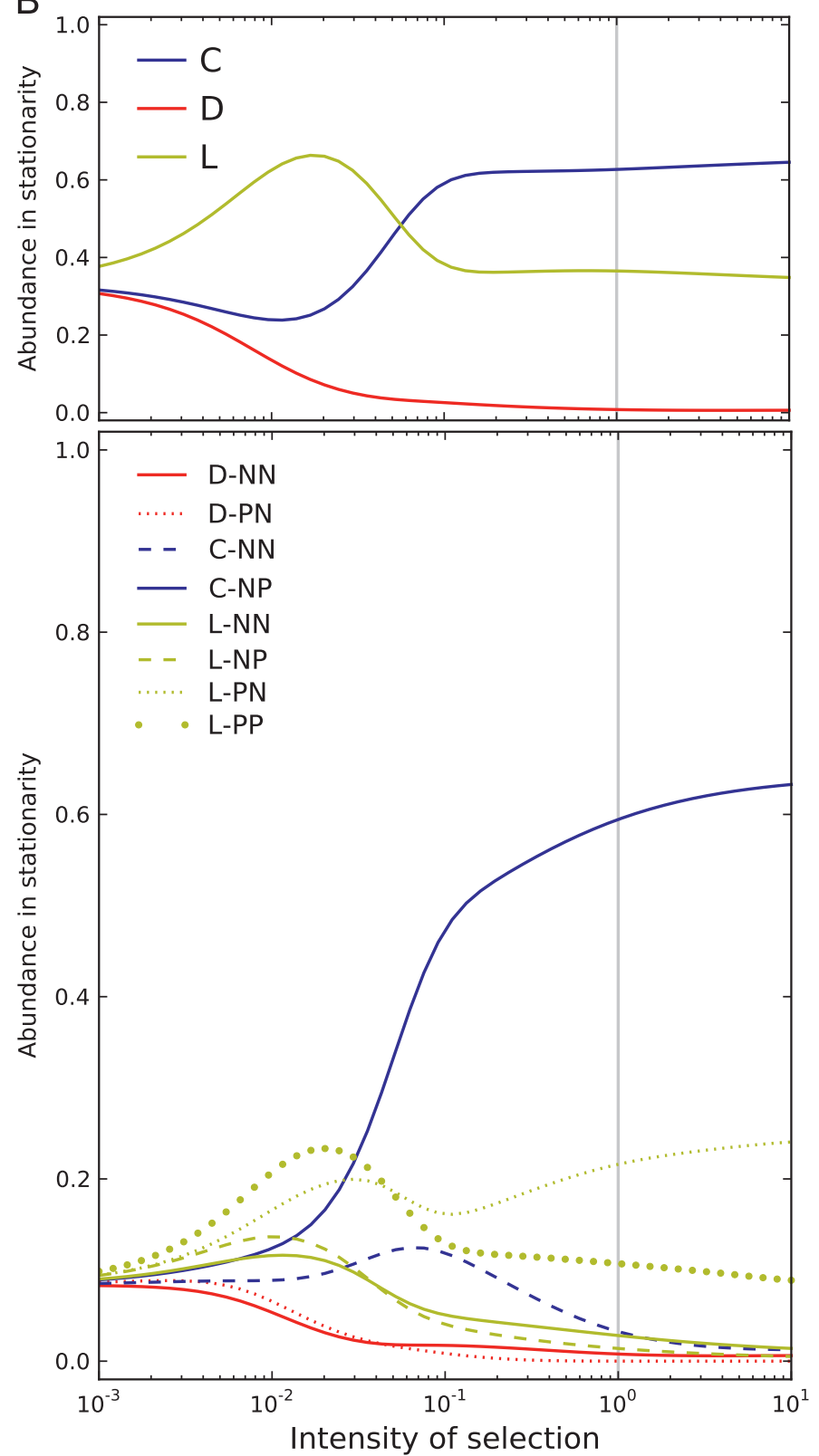

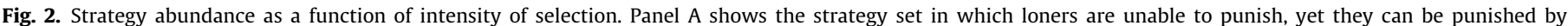

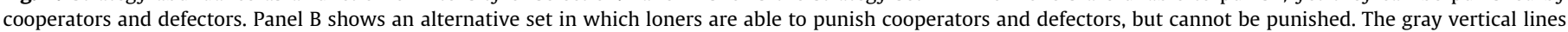

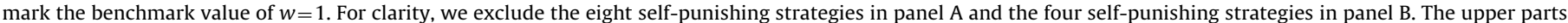
depict strategies grouped by contribution in the first stage of the game. Parameters are as follows: $M=100, n=5, r=3, \gamma=0.3, \beta=\sigma=c=1$. 
where again the limit is for $M \rightarrow \infty$. In this case, $x_{C-N P}=\frac{2}{3}$, $x_{L-P N}=\frac{1}{4}$, and $x_{L-P P}=\frac{1}{12}$. All other strategies have abundance zero. Cooperators who punish defectors are the most popular strategy at an abundance of $\frac{2}{3}$.

\section{Discussion}

The key issue in this kind of models is that cooperators who punish defectors can always be invaded by cooperators who do not punish at all-punishment is destabilized by those who cooperate unconditionally and never punish. These non-punishing cooperators can be invaded by defective strategies. Whether cooperation is favored or not in the long run depends on the role of loners in bringing defective populations back into cooperation. This catalyzing role of loners critically depends on them not being punished. Loners fail to outcompete defectors only when they are punished by others, and the bridge towards coercion via freedom is compromised.

Whether cooperators who punish defectors prevail in the optional public goods game depends strongly on how loners interact with other strategies. Concerning this point, Rand and Nowak assert that their model requires some "implicit mechanism of gossip" by which loners are informed of the actions of the participants in the game. We note that on top of that, those excluded would still need to be able to exert their punishment power on those who play the game. There may be settings where such information transfers, and mechanisms of exerting power "in absence" are not possible or compromised. This depends specifically on the situation being modeled. If the game is such that loners are truly alone and isolate themselves (e.g., "collecting mushrooms instead of participating in a collective hunt"), costly punishment by cooperators towards defectors prevails, and beats both, antisocial punishment as well as defection. If loners can be punished, they fail to serve as a stepping stone for cooperative strategies to invade defectors.

We therefore conclude that with loners who are truly alone, and thus excluded from being punished, the combination of voluntary participation and punishment does lead to the evolution of cooperation, in spite of antisocial punishment being an option. Only in situations in which loners are not isolated and can be punished by others, neither cooperation nor punishment evolve. Instead, a mixture of cooperative, uncooperative and non-participant strategies that either punish or not turn out to be equally prevalent, essentially independent of the intensity of selection.

In summary, allowing the punishment of those who abstain compromises the freedom that comes with voluntary participation, and disrupts the path towards successful collective endeavors.

\section{Acknowledgement}

We thank C. Hauert, C. Hilbe, M. Milinski, M. van Veelen and K. Sigmund for discussions. Financial support by the MPG is gratefully acknowledged.

\section{Appendix A. Transition probabilities in the Moran process}

To study the evolutionary dynamics we use the Moran process (Moran, 1962). It allows for studying the interplay between selection and mutation under demographic noise. The Moran process considers a finite population of constant size $M$. At every time step one strategy is chosen for reproduction in proportion to its performance in the current population. A copy of this strategy is added to the population after removing a random strategy. With a small probability, the strategy that is copied changes its type to any of the other available strategies. This process results in an ergodic Markov chain (Fudenberg and Imhof, 2006).

The fitness of strategy $A$ is given by $f_{A}=\exp \left(w \pi_{A}\right)$, where $\pi_{A}$ is the payoff from the game and $w$ is the intensity of selection. The payoffs are given in the supplementary material of Rand and Nowak (2011). The fixation probability of a single mutant playing strategy $A$ in a population of $M-1$ individuals playing $B$ is given by

$$
\phi_{A}^{B}=\frac{1}{1+\sum_{k=1}^{M-1} \prod_{j=1}^{k} \frac{T_{j}^{-}}{T_{j}^{+}}}
$$

where

$$
T_{i}^{+}=\frac{i f_{A}}{i f_{A}+(M-1) f_{B}} \frac{M-i}{M}
$$

and

$T_{i}^{-}=\frac{(M-i) f_{B}}{i f_{A}+(M-1) f_{B}} \frac{i}{M}$

Here $M$ is the size of the population. For a sufficiently small probability of mutation $(\mu \ll 1)$, the transition probability from a homogeneous population of $A$ individuals into a homogeneous population of $B$ individuals is given by

$\Phi_{A}^{B}=\frac{\mu}{M-1} \phi_{B}^{A}$

The Markov chain between homogeneous populations is thus given by a matrix $A=\left[A_{i j}=\Phi_{i}^{j}\right]$, for $i, j$ in $1,2, \ldots, s$; where $s$ is the number of strategies, and $i \neq j$. Matrix $A$ is normalized such that the rows sum up to 1 . The abundances are given by the eigenvector corresponding to eigenvalue 1 . In the strong selection limit, we let $w \rightarrow \infty$ such that $\Phi_{i}^{j}$ is $0,1, \frac{1}{2}$ or $\frac{1}{M}$. Throughout the paper we plot the stationary distribution as a function of the intensity of selection (continuous lines). To check that the theoretical prediction is accurate we use Monte Carlo simulations (symbols in the Figures). The stationary distribution is estimated by averaging the result of 1500 runs. Each run is composed of $6 \times 10^{6}$ generations, starting in a random population. The average

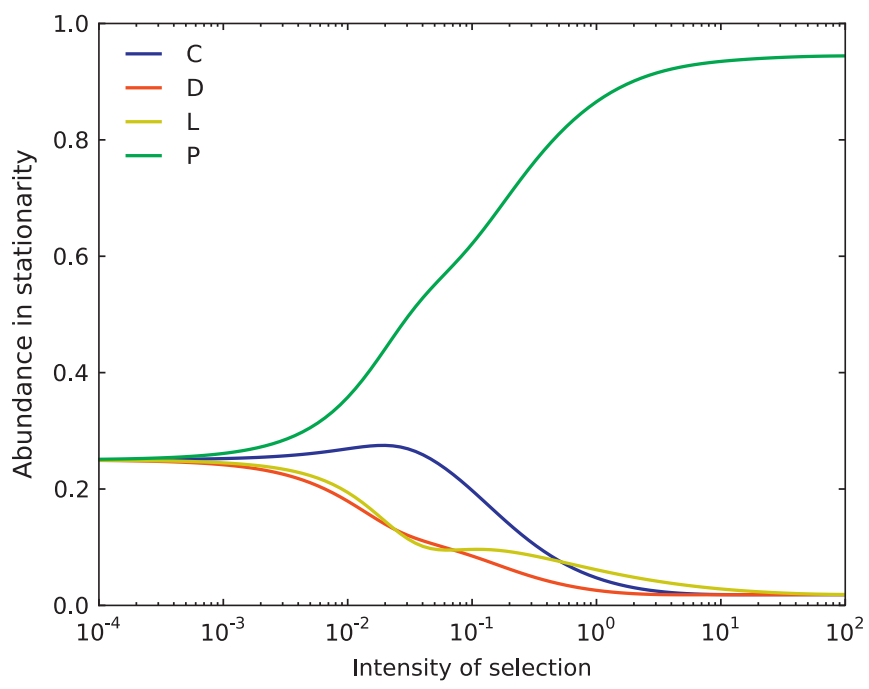

Fig. B1. Abundance in stationarity as a function of the intensity of selection $(M=100, n=5, r=3, \gamma=0.3, \beta=\sigma=c=1)$. 
considers the composition of the population during the last $20 \%$ generations of each run. The mutation rate is set to $10^{-4}$.

\section{Appendix B. Restricted strategy set}

Here we compute the results of Hauert et al. (2007) using exponential payoff to fitness mapping, as discussed in Appendix A. The stationary distribution for strong selection is given by

$$
\left(\begin{array}{c}
x_{C} \\
x_{D} \\
x_{L} \\
x_{P}
\end{array}\right)=\frac{1}{8+M}\left(\begin{array}{c}
2 \\
2 \\
2 \\
2+M
\end{array}\right) \rightarrow\left(\begin{array}{l}
0 \\
0 \\
0 \\
1
\end{array}\right)
$$

where the limit is for $M \rightarrow \infty$. Fig. B1 shows the frequencies in stationarity as a function of the intensity of selection. In the limit of rare mutation $(\mu \rightarrow 0)$, infinite selection intensity $(w \rightarrow \infty)$ and infinite population size $(M \rightarrow \infty)$, the support of the stationary distribution is the monomorphic state of cooperators who punish defectors.

\section{Appendix C. Strategy sets for partial isolation of loners}

The strategy sets for the case of partial isolation of loners are as follows:

Table C1

Partial isolation-loners can be punished, but cannot punish others.

\begin{tabular}{ll}
\hline$D-N N N$ & Defectors who do not punish \\
$D-N N P$ & Defectors who only punish loners \\
$D-N P N$ & Defectors who only punish other defectors \\
$D-N P P$ & Defectors who punish loners and other defectors \\
$D-P N N$ & Defectors who only punish cooperators \\
$D-P N P$ & Defectors who punish cooperators and loners \\
$D-P P N$ & Defectors who punish cooperators and other defectors \\
$D-P P P$ & Defectors who punish everyone else \\
$C-N N N$ & Cooperators that do not punish \\
$C-N N P$ & Cooperators who only punish loners \\
$C-N P N$ & Cooperators who only punish defectors \\
$C-N P P$ & Cooperators who punish defectors and loners \\
$C-P N N$ & Cooperators who only punish other cooperators \\
$C-P N P$ & Cooperators who punish loners and other cooperators \\
$C-P P N$ & Cooperators who punish defectors and other cooperators \\
$C-P P P$ & Cooperators who punish everyone else \\
$L$ & Loners who do not punish anyone \\
\hline
\end{tabular}

Table C2

Partial isolation-loners cannot be punished, but can punish others.

\begin{tabular}{ll}
\hline$D-N N$ & Defectors who do not punish \\
$D-N P$ & Defectors who only punish other defectors \\
$D-P N$ & Defectors who only punish cooperators \\
$D-P P$ & Defectors who punish cooperators and other defectors \\
$C-N N$ & Cooperators who do not punish \\
$C-N P$ & Cooperators who only punish defectors \\
$C-P N$ & Cooperators who only punish other cooperators \\
$C-P P$ & Cooperators who punish defectors and other cooperators \\
$L-N N$ & Loners who do not punish \\
$L-N P$ & Loners who only punish defectors \\
$L-P N$ & Loners who only punish cooperators \\
$L-P P$ & Loners who punish cooperators and defectors \\
\hline
\end{tabular}

\section{References}

Boyd, R., Gintis, H., Bowles, S., Richerson, P., 2003. The evolution of altruistic punishment. Proc. Natl. Acad. Sci. USA 100, 3531-3535.

Brandt, H., Hauert, C., Sigmund, K., 2006. Punishing and abstaining for public goods. Proc. Natl. Acad. Sci. USA 103 (2), 495-497.

De Silva, H., Hauert, C., Traulsen, A., Sigmund, K., 2010. Freedom, enforcement, and the social dilemma of strong altruism. J. Evol. Econ. 20, 203-217.

Dreber, A., Rand, D.G., 2012. Retaliation and antisocial punishment are overlooked in many theoretical models as well as behavioral experiments. Behav. Brain Sci. 35 (1), 24

Fehr, E., Gächter, S., 2000. Cooperation and punishment in public goods experiments. Am. Econ. Rev. 90, 980-994.

Fehr, E., Gachter, S., 2002. Altruistic punishment in humans. Nature 415 (6868), 137-140.

Fowler, J.H., 2005. Altruistic punishment and the origin of cooperation. Proc. Natl. Acad. Sci. USA 102 (19), 7047-7049.

Fudenberg, D., Imhof, L.A., 2006. Imitation processes with small mutations. J. Econ. Theory 131, 251-262.

Hauert, C., De Monte, S., Hofbauer, J., Sigmund, K., 2002. Volunteering as red queen mechanism for cooperation in public goods games. Science 296, 1129-1132.

Hauert, C., Traulsen, A., Brandt, H., Nowak, M.A., Sigmund, K., 2007. Via freedom to coercion: the emergence of costly punishment. Science 316, 1905-1907.

Hauert, C., Traulsen, A., Brandt, H., Nowak, M.A., Sigmund, K., 2008. Public goods with punishment and abstaining in finite and infinite populations. Biol. Theory 3, 114-122.

Helbing, D., Szolnoki, A., Perc, M., Szabó, G., 2010. Evolutionary establishment of moral and double moral standards through spatial interactions. PLoS Comput. Biol. 6, e1000758.

Henrich, J., McElreath, R., Barr, A., Ensminger, J., Barrett, C., Bolyanatz, A., Cardenas, J.C., Gurven, M., Gwako, E., Henrich, N., Lesorogol, C., Marlowe, F., Tracer, D., Ziker, J., 2006. Costly punishment across human societies. Science 312, 1767-1770.

Herrmann, B., Thöni, C., Gächter, S., 2008. Antisocial punishment across societies. Science 319, 1362-1367.

Johnson, T., Dawes, C., Fowler, J., McElreath, R., Smirnov, O., 2009. The role of egalitarian motives in altruistic punishment. Econ. Lett. 102 (3), 192-194.

Mathew, S., Boyd, R., 2009. When does optional participation allow the evolution of cooperation? Proc. R. Soc. Lond. B 276, 1167-1174.

Moran, P.A.P., 1962. The Statistical Processes of Evolutionary Theory. Clarendon Press, Oxford.

Rand, D.G., Armao IV, J.J., Nakamaru, M., Ohtsuki, H., 2010. Anti-social punishment can prevent the co-evolution of punishment and cooperation. J. Theor. Biol. 265 (4), 624-632.

Rand, D.G., Nowak, M.A., 2011. The evolution of antisocial punishment in optional public goods games. Nat. Commun. 2, 434.

Sigmund, K., 2007. Punish or perish? Retaliation and collaboration among humans. Trends Ecol. Evol. 22 (11), 593-600.

Sigmund, K., 2010. The Calculus of Selfishness. Princeton University Press.

Traulsen, A., Hauert, C., De Silva, H., Nowak, M.A., Sigmund, K., 2009. Exploration dynamics in evolutionary games. Proc. Natl. Acad. Sci. USA 106, 709-712.

Traulsen, A., Shoresh, N., Nowak, M.A., 2008. Analytical results for individual and group selection of any intensity. Bull. Math. Biol. 70, 1410-1424.

Yamagishi, T., 1986. The provision of a sanctioning system as a public good. J. Pers. Soc. Psychol. 51, 110-116. 